\title{
STOCHASTIC ANALYSIS OF THE DEPARTURE AND QUASI-INPUT PROCESSES IN A VERSATILE SINGLE-SERVER QUEUE
}

\author{
J.R. ARTALEJO and A. GOMEZ-CORRAL \\ University Complutense of Madrid, Mathematics Faculty \\ Department of Statistics and O.R., 28040 Madrid, Spain
}

(Received July, 1995; Revised October, 1995)

\begin{abstract}
This paper is concerned with the stochastic analysis of the departure and quasi-input processes of a Markovian single-server queue with negative exponential arrivals and repeated attempts. Our queueing system is characterized by the phenomenon that a customer who finds the server busy upon arrival joins an orbit of unsatisfied customers. The orbiting customers form a queue such that only a customer selected according to a certain rule can reapply for service. The intervals separating two successive repeated attempts are exponentially distributed with rate $\alpha+j \mu$, when the orbit size is $j \geq 1$. Negative arrivals have the effect of killing some customer in the orbit, if one is present, and they have no effect otherwise. Since customers can leave the system without service, the structural form of type $\mathrm{M} / \mathrm{G} / 1$ is not preserved. We study the Markov chain with transitions occurring at epochs of service completions or negative arrivals. Then we investigate the departure and quasi-input processes.
\end{abstract}

Key words: Queueing, Repeated Attempts, Negative Arrivals, Regenerative Processes, Generalized Hypergeometric Functions.

AMS (MOS) subject classifications: $90 \mathrm{~B} 22,60 \mathrm{~K} 25$.

\section{Introduction}

Queueing systems with repeated attempts have wide practical use in designing packet switching networks and telecommunication systems. The main characteristic of a single-server queue with repeated attempts is that a customer who finds the server busy upon arrival is obliged to leave the service area, but some time later he comes back to re-initiate his demand. Between trials a customer is said to be "in orbit". Most papers assume that each orbiting customer has probability $\mu d t+o(d t)$ of re-applying for service in $(t, t+d t)$, independently of each other customer in orbit at time $t$. In what follows, we call this retrial policy as classical retrial discipline. For a review of the literature on this topic see [4]. Nevertheless, there are other types of queueing situations, in which the retrial rate is independent of the number of customers (if any) in orbit i.e., the retrial rate is $\alpha$ if the orbit is not empty at time $t$ and zero if the orbit is empty. This second retrial policy will be called constant retrial discipline. A detailed discussion of situations where such discipline arises can be found in [5]. In related work, Martin and Artalejo [7] examined the $\mathrm{M} / \mathrm{G} / 1$ with two types of customers and constant retrial discipline. 
Recently, Harrison and Pitel [6] studied an M/M/1 queue with "negative" arrivals. Any arriving customer joins the system with the intention of getting served and then leaving the system. They are treated in the normal way by the server and are taken from the queue according to a specified queueing discipline. At a negative arrival epoch, the system is only affected if customers are present. Then, a customer is removed from the system i.e., each negative arrival reduces the total customers count by one unit. Several practical applications justify the study of these queueing models. In multiprocessor computer systems, negative arrivals represent commands to delete some transaction. In neural networks, primary and negative arrivals represent excitatory and inhibitory signals, respectively.

The purpose of the present work is to study the departure and quasi-input processes of a versatile single-server queue allowing the simultaneous presence of classical and constant repeated attempts, and negative arrivals. Our queueing model was introduced in [1], where the classification of states, stationary distribution, waiting time and busy period were studied. Thus, this paper completes the investigation initialed in [1].

To allow the presence of both types of repeated attempts we will assume that intervals between two successive repeated attempts are exponentially distributed with rate $\alpha\left(1-\delta_{0 j}\right)+j \mu$, when the orbit size is $j . \delta_{0 j}$ denotes Kronecker's function. This retrial policy will be called linear retrial discipline. The main characteristic of our model is its versatility so, in section 2 , we discuss concrete interpretations of the systems associated with some specific choices of the parameters. Nevertheless, the queueing system under consideration has also an intrinsic interest to model some situations in packet switching networks. Consider a computer network which consists of a group of processors connected with a central transmission unit $(C T U)$. If a processor wishes to send a message it first sends the message to the $C T U$. If the transmission medium is available, the $C T U$ sends immediately the message; otherwise the message will be stored in a buffer and the $C T U$ must retry the transmission some time later. For mathematical convenience we assume that this random time is exponentially distributed; then we construct a retrial rate of the simplest possible type by assuming that there are two contributions to the retrial intensity. The first one $\alpha$ is fixed and intrinsic with the network design, whereas the second one $j \mu$ depends on the number of units in the buffer. In addition, the $C T U$ sends negative signals to the buffer in order to remove one of the unsatisfied units. This mechanism guarantees a moderate level of internal congestion in the buffer.

It should be pointed out that the presence of a stream of negative arrivals has a profound influence on the system. This is revealed by the fact that the structural form of the M/G/1 queue is not preserved. We also observe that, due to the existence of negative arrivals, the limiting probability distribution of the continuous time process describing the number of customers in the system is not equal to the distribution of the embedded process describing the state of the system just after service completion epochs.

The rest of the paper is organized as follows. In section 2 we describe the mathematical model. The study of the embedded Markov chains at service completion and killing epochs and their corresponding factorial moments are carried out in section 3 . In section 4 we obtain the Laplace-Stieltjes transform of the interdeparture times and its factorial moments. The quasiinput process is analyzed in section 5 and, finally, some conclusions are given in section 6 .

\section{Description of the Mathematical Model}

We consider a single-server queueing system with two independent Poisson streams with rates $\lambda>0$ and $\delta \geq 0$, corresponding to primary and negative arrivals, respectively. If the server is free, an arriving primary customer begins to be served and leaves the system after service com- 
pletion. Any primary customer finding the server busy upon arrival must leave the service area immediately and seek service again at subsequent epochs until he finds the server free. He is then said to be "in orbit". These unsatisfied customers form a pool such that only one customer selected according to a certain rule can access to the server. The time intervals describing the repeated attempts are assumed to be independent and exponentially distributed with rate $\alpha\left(1-\delta_{0 j}\right)+j \mu$, when there are $j$ customers in orbit. Negative arrivals have the effect of deleting one customer of the orbit, if there are any, who is selected according to some specified killing strategy. Service times are independent exponential random variables with rate $\nu$. The streams of primary and negative arrivals, intervals separating successive repeated attempts and service times are assumed to be mutually independent.

We now discuss some particularizations which can be obtained with specific choices of the parameters $\alpha, \mu$ and $\delta$. First, the case $\delta=0$ leads to a retrial queue with linear retrial discipline which generalizes the classical and constant retrial queues described in the literature. The case $\mu=0$ and $\delta=(1-H) \alpha, H \in(0,1)$, corresponds to the constant retrial queue where the customer at the head of the orbit is nonpersistent. Finally, the single-server Markovian queue with classical waiting line and negative arrivals is obtained by letting $\alpha \rightarrow+\infty$ and/or $\mu \rightarrow+\infty$.

The state of the system at time $t$ can be described by the bivariate process $X=\{X(t), t \geq 0\}=\{(C(t), Q(t)), t \geq 0\}$, where $Q(t)$ represents the number of customers in orbit and $C(t)$ is equal to 1 or 0 according as the server is busy or free, respectively. Note that the process $X$ takes values on the semi-strip $S=\{0,1\} \times \mathbb{N}$.

We shall consider that the process $X$ is in the ergodic case, which exists if and only if one of the following conditions is verified (see [1])

(C1) $\quad \mu=0$ and $\gamma<1$,

(C2) $\mu>0$ and $\rho<1$, where

$$
\gamma=\frac{(\lambda-\delta)(\lambda+\alpha)}{\nu \alpha} \text { and } \rho=\frac{\lambda}{\delta+\nu}
$$

We also define the limiting probabilities

$$
P_{i j}=\lim _{t \rightarrow+\infty} P\{C(t)=i, Q(t)=j\}, \text { for }(i, j) \in S,
$$

which are positive if and only if $(C 1)$ or $(C 2)$ is satisfied. The probabilities $P_{i j}$ and the partial generating functions defined as $P_{i}(z)=\sum_{j=0}^{\infty} P_{i j} z^{j}$, for $i \in\{0,1\}$, can be expressed in terms of hypergeometric series (see [1]). Since our model has a Poisson input, the stationary distribution is equal to the equilibrium state distribution just prior to arrival epochs.

Finally, we introduce some notation used in the rest of the paper. Let us consider the generalized hypergeometric series defined as follows

$$
k+1 F_{k}\left(\begin{array}{c}
a_{1}, a_{2}, \ldots, a_{k+1} ; z \\
b_{1}, b_{2}, \ldots, b_{k}
\end{array}\right)=\sum_{n=0}^{\infty} \frac{\left(a_{1}\right)_{n}\left(a_{2}\right)_{n} \ldots\left(a_{k+1}\right)_{n}}{\left(b_{1}\right)_{n}\left(b_{2}\right)_{n} \ldots\left(b_{k}\right)_{n}} \frac{z^{n}}{n !}
$$

where $(x)_{n}$ is the Pochhammer symbol

$$
(x)_{n}=\left\{\begin{array}{cc}
1, & \text { for } n=0, \\
x(x+1) \ldots(x+n-1), & \text { for } n \geq 1 .
\end{array}\right.
$$

As is usual, we denote ${ }_{2} F_{1}\left(\begin{array}{c}a_{1}, a_{2} ; z \\ b_{1}\end{array}\right)$ by $F\left(a_{1}, a_{2} ; b_{1} z\right)$. 


\section{Embedded Markov Chain at Service Completion and Killing Epochs}

In this section, we consider the system at epochs $\eta_{n}$ of service completion or negative arrival. Our purpose is to study the Markov chain with transitions occurring at these instants.

First, we define

$$
\Pi_{j}=\lim _{n \rightarrow+\infty} P\left\{C\left(\eta_{n}+0\right)+Q\left(\eta_{n}+0\right)=j\right\}, \text { for } j \geq 0 .
$$

The above probabilities can be expressed as

where

$$
\Pi_{j}=\left\{\begin{array}{cc}
\bar{\Pi}_{0}, & \text { for } j=0, \\
\bar{\Pi}_{j}+\widetilde{\Pi}_{j}, & \text { for } j \geq 1,
\end{array}\right.
$$

$$
\begin{gathered}
\bar{\Pi}_{j}=\lim _{n \rightarrow+\infty} P\left\{X\left(\eta_{n}+0\right)=(0, j)\right\}, \quad \text { for } j \geq 0, \\
\widetilde{\Pi}_{j}=\lim _{n \rightarrow+\infty} P\left\{X\left(\eta_{n}+0\right)=(1, j-1)\right\}, \quad \text { for } j \geq 1 .
\end{gathered}
$$

Note that with the help of $\bar{\Pi}_{j}$ and $\widetilde{\Pi}_{j}$, we can distinguish if a transition of our Markov chain corresponds to a service completion or a negative arrival.

It is well-known that any stochastic process whose sample functions are (almost all) step functions with unit jumps has the same limiting distribution just prior to its points of increase as it does just after its points of decrease, when this limiting distribution exists (see [3], pp. 187). Thus, the limiting probabilities $P_{j}$ associated with the total number of customers in the system satisfy

$$
P_{j}=P_{0 j}+P_{1, j-1}\left(1-\delta_{0 j}\right)=\Pi_{j}, \quad \text { for } j \geq 0 \text {. }
$$

We assume in the rest of this section that at epoch $t=0$, the system is in state $(0,0)$. We define a busy period, $L$, as the period that starts at epoch when a primary customer enters an empty system and ends at the next service completion epoch at which the system becomes empty. A regeneration cycle, $T$, is defined as the first-passage time from state $(0,0)$ to state $(0,0)$. It should be noted that the continuous time process $\{X(t), t \geq 0\}$ is a regenerative process with embedded renewal process $\left\{T_{1}, T_{2}, \ldots\right\}$, where $T_{i}$ denotes the $i$ th cycle. Moreover, $\left\{X_{n}\right\}_{n>0}=$ $\left\{\left(C\left(\eta_{n}+0\right), Q\left(\eta_{n}+0\right)\right)\right\}_{n}>0$ is a discrete time regenerative process with renewal sequence $\left\{N_{1}, N_{2}, \ldots\right\}$, where $N_{i}$ is the number of generalized departures (i.e., customers served or deleted by a negative arrival) during the $i$ th cycle, $T_{i}$. For convenience of notation, we shall denote $T=T_{1}$ and $N=N_{1}$.

With these preliminaries we are ready to state the following result in which we study the distributions of the embedded Markov chains at service completion and killing epochs.

Theorem 1: If the process $\{X(t), t \geq 0\}$ is ergodic, then

i) The probability distribution of the number of customers in orbit just after a service completion epoch is given by

$$
\begin{gathered}
\bar{\pi}_{0}=\left(F\left(1,1+\frac{\lambda+\alpha}{\mu} ; 1+\frac{\alpha+\delta \rho}{\mu} ; \rho\right)\right)^{-1}, \\
\bar{\pi}_{j}=\bar{\pi}_{0} \frac{\left(1+\frac{\lambda+\alpha}{\mu}\right)_{j}}{\left(1+\frac{\alpha+\delta \rho}{\mu}\right)_{j}} \rho^{j}, \text { for } j \geq 1 .
\end{gathered}
$$


ii) If $\delta>0$, the probability distribution of the number of customers in the system just after a killing epoch is given by

where

$$
\tilde{\pi}_{j}=A \frac{\delta\left(1+\frac{\lambda+\alpha}{\mu}\right)_{j}}{\nu\left(1+\frac{\alpha+\delta \rho}{\mu}\right)_{j}} \rho^{j}, \quad \text { for } j \geq 1
$$

$$
A=\left(\frac{\delta(\lambda+\alpha+\mu)}{\nu(\alpha+\mu+\delta \rho)} \rho F\left(1,2+\frac{\lambda+\alpha}{\mu} ; 2+\frac{\alpha+\delta \rho}{\mu} ; \rho\right)\right)^{-1}
$$

Proof: By a basic result in the theory of regenerative processes (see [8]), we have that the probabilities $\bar{\Pi}_{j}$ and $\widetilde{\Pi}_{j}$ can be expressed as follows

$$
\begin{gathered}
\bar{\Pi}_{j}=\frac{E\left[\bar{N}^{j}\right]}{E[N]}, \text { for } j \geq 0, \\
\tilde{\Pi}_{j}=\frac{E\left[\tilde{N}^{j}\right]}{E[N]}, \text { for } j \geq 1,
\end{gathered}
$$

where $\bar{N}^{j}$ (respectively, $\tilde{N}^{j}$ ) is the number of service completion (respectively, killing) epochs at which $j$ customers are left behind in the system during the first regenerative cycle $(0, T]$. Since $\bar{N}^{0}=1$, then $\bar{\Pi}_{0}=1 / E[N]$.

For convenience we denote $(0, j)$ by $A_{j}$ and $(1, j)$ by $B_{j}$, for $j \geq 0$. We also consider $\bar{N}_{a b}^{j}$ (respectively, $\tilde{N}_{a b}^{j}$ ) as the number of service completion (respectively, killing) epochs at which $j$ customers are left behind in the system during the first-passage time, $T_{a b}$, from state $a$ to state $b$. Then, $\bar{N}^{j}=\bar{N}_{B_{0} A_{0}}^{j}$ and $\widetilde{N}^{j}=\widetilde{N}_{B_{0} A_{0}}^{j}$, for $j \geq 1$.

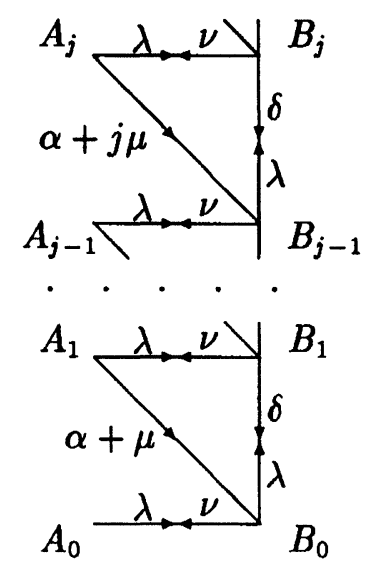

Figure 1. State space and transitions

Let $\Phi_{a b}^{j}(x, y)$ be the generating function of the pair $\left(\bar{N}_{a b}^{j}, \tilde{N}_{a b}^{j}\right)$ defined by

$$
\Phi_{a b}^{j}(x, y)=E\left[x^{\bar{N}_{a b}^{j}} \tilde{N}_{a b}^{j}\right], \text { for } j \geq 1,|x| \leq 1 \text { and }|y| \leq 1 .
$$


Taking into account that a direct transition from the state $B_{0}$ to state $A_{0}$ (respectively, from $B_{0}$ to $B_{1}$ ) occurs with probability $\nu(\lambda+\nu)^{-1}$ (respectively, $\lambda(\lambda+\nu)^{-1}$ ), we get that $\Phi_{B_{0}}^{j} A_{0}(x, y)$ can be written as follows

$$
\Phi_{B_{0} A_{0}}^{j}(x, y)=\frac{\nu}{\lambda+\nu}+\frac{\lambda}{\lambda+\nu} \Phi_{B_{1} A_{0}}^{j}(x, y)
$$

These arguments can be extended, with the help of Figure 1, to derive the following set of equations

$$
\begin{gathered}
\Phi_{B_{1} A_{0}}^{j}(x, y)=\Phi_{B_{1} B_{0}}^{j}(x, y) \Phi_{B_{0} A_{0}}^{j}(x, y), \\
\Phi_{B_{i} B_{i-1}}^{j}(x, y)=\frac{\delta}{\lambda+\delta+\nu}\left(1-\delta_{i j}+y \delta_{i j}\right)+\frac{\lambda}{\lambda+\delta+\nu} \Phi_{B_{i+1} B_{i-1}}^{j}(x, y) \\
+\frac{\nu}{\lambda+\delta+\nu}\left(1-\delta_{i j}+x \delta_{i j}\right) \Phi_{A_{i} B_{i-1}}^{j}(x, y), \\
\Phi_{B_{i+1} B_{i-1}}^{j}(x, y)=\Phi_{B_{i+1} B_{i}}^{j}(x, y) \Phi_{B_{i} B_{i-1}}^{j}(x, y), \\
\Phi_{A_{i} B_{i-1}}^{j}(x, y)=\frac{\alpha+i \mu}{\lambda+\alpha+i \mu}+\frac{\lambda}{\lambda+\alpha+i \mu} \Phi_{B_{i} B_{i-1}}^{j}(x, y),
\end{gathered}
$$

for $i \geq 1, j \geq 1,|x| \leq 1$ and $|y| \leq 1$. obtain

Differentiating the set of equations (3.6)-(3.10) with respect to $x$ and setting $x=y=1$ we

$$
\begin{aligned}
& E\left[\bar{N}_{B_{0} A_{0}}^{j}\right]=\frac{\lambda}{\lambda+\nu} E\left[\bar{N}_{B_{1} A_{0}}^{j}\right] \\
& E\left[\bar{N}_{B_{1} A_{0}}^{j}\right]=E\left[\bar{N}_{B_{1} B_{0}}^{j}\right]+E\left[\bar{N}_{B_{0} A_{0}}^{j}\right] \\
& E\left[\bar{N}_{B_{i} B_{i-1}}^{j}\right]=\frac{\lambda}{\lambda+\delta+\nu} E\left[\bar{N}_{B_{i+1} B_{i-1}}^{j}\right]+\frac{\nu}{\lambda+\delta+\nu}\left(\delta_{i j}+E\left[\bar{N}_{A_{i} B_{i-1}}^{j}\right]\right) \\
& E\left[\bar{N}_{B_{i+1} B_{i-1}}^{j}\right]=E\left[\bar{N}_{B_{i+1} B_{i}}^{j}\right]+E\left[\bar{N}_{B_{i} B_{i-1}}^{j}\right] \\
& E\left[\bar{N}_{A_{i} B_{i-1}}^{j}\right]=\frac{\lambda}{\lambda+\alpha+i \mu} E\left[\bar{N}_{B_{i} B_{i-1}}^{j}\right],
\end{aligned}
$$

for $i \geq 1$ and $j \geq 1$. From (3.11) and (3.12) we find that

$$
E\left[\bar{N}^{j}\right]=\frac{\lambda}{\nu} E\left[\bar{N}_{B_{1} B_{0}}^{j}\right], \text { for } j \geq 1
$$

Analogously, from (3.13)-(3.15) we get

$$
E\left[\bar{N}_{B_{i} B_{i-1}}^{j}\right]=\frac{\lambda\left(i+\frac{\lambda+\alpha}{\mu}\right)}{(\delta+\nu)\left(i+\frac{\alpha+\delta \rho}{\mu}\right)}\left(\frac{\nu}{\lambda} \delta_{i j}+E\left[\bar{N}_{B_{i+1} B_{i}}^{j}\right), \quad \text { for } i \geq 1 \text { and } j \geq 1\right. \text {. }
$$

Thus the following expression for $E\left[\bar{N}^{j}\right]$ is easily obtained

$$
E\left[\bar{N}^{j}\right]=\frac{\left(1+\frac{\lambda+\alpha}{\mu}\right)_{j}}{\left(1+\frac{\alpha+\delta \rho}{\mu}\right)_{j}}, \quad \text { for } j \geq 1 .
$$


Then the expected number of service completions occurring during a cycle is given by

$$
E[\bar{N}]=F\left(1,1+\frac{\lambda+\alpha}{\mu} ; 1+\frac{\alpha+\delta \rho}{\mu} ; \rho\right) .
$$

Applying a similar scheme for the expected number of customers deleted during $(0, T]$, we deduce the following expressions

$$
E\left[\tilde{N}^{j}\right]=\frac{\delta\left(1+\frac{\lambda+\alpha}{\mu}\right)_{j}}{\nu\left(1+\frac{\alpha+\delta \rho}{\mu}\right)_{j}} \rho^{j}, \quad \text { for } j \geq 1
$$

Taking into account that the expected number of killing customers in a cycle can be expressed as $E[\tilde{N}]=\sum_{j=1}^{\infty} E\left[\tilde{N}^{j}\right]$, it follows from (3.17) that

$$
E[\tilde{N}]=\frac{\delta(\lambda+\alpha+\mu)}{\nu(\alpha+\mu+\delta \rho)} \rho F\left(1,2+\frac{\lambda+\alpha}{\mu} ; 2+\frac{\alpha+\delta \rho}{\mu} ; \rho\right) .
$$

Thus, $E[N]$ is

$$
E[N]=1+\frac{\lambda(\lambda+\alpha+\mu)}{\nu(\alpha+\mu+\delta \rho)} F\left(1,2+\frac{\lambda+\alpha}{\mu} ; 2+\frac{\alpha+\delta \rho}{\mu} ; \rho\right)
$$

Since

$$
\begin{aligned}
& \bar{\pi}_{j}=\frac{\bar{\Pi}_{j}}{\sum_{k=0}^{\infty} \bar{\Pi}_{k}}, \quad \text { for } j \geq 0, \\
& \tilde{\pi}_{j}=\frac{\tilde{\Pi}_{j}}{\sum_{k=1}^{\infty} \tilde{\Pi}_{k}}, \quad \text { for } j \geq 1,
\end{aligned}
$$

it follows from (3.4), (3.5), (3.16), (3.17) and (3.19)-(3.21) that formulae (3.1)-(3.3) are correct.

To conclude, we observe that the convergence of the series involved in this theorem follows from an easy application of ratio test and Raabe's test. $\left\{\tilde{\pi}_{j}\right\}_{j \geq 1}$.

Our second goal in this section is to find the factorial moments of the sequences $\left\{\bar{\pi}_{j}\right\}_{j \geq 0}$ and

Theorem 2: If the process $\{X(t), t \geq 0\}$ is ergodic, then

i) The kth factorial moment of the embedded Markov chain at service completion epochs, $\bar{M}_{k}$, is given by

$$
\bar{M}_{k}=\bar{\pi}_{0} \frac{\left(1+\frac{\lambda+\alpha}{\mu}\right)_{k}}{\left(1+\frac{\alpha+\delta \rho}{\mu}\right)_{k}} \rho^{k} k ! F\left(k+1, k+1+\frac{\lambda+\alpha}{\mu} ; k+1+\frac{\alpha+\delta \rho}{\mu} ; \rho\right), \text { for } k \geq 0,
$$

where $\bar{\pi}_{0}$ was given in equation (3.1).

ii) The kth factorial moment of the embedded Markov chain at killing epochs, $\widetilde{M}_{k}$, is given $\tilde{M}_{k}=\frac{\delta}{\nu E(\tilde{N})}\left(\frac{\left(1+\frac{\lambda+\alpha}{\mu}\right)_{k}}{\left(1+\frac{\alpha+\delta \rho}{\mu}\right)_{k}} \rho^{k} k ! F\left(k+1, k+1+\frac{\lambda+\alpha}{\mu} ; k+1+\frac{\alpha+\delta \rho}{\mu} ; \rho\right)-\delta_{0 k}\right)$,

for $k \geq 0$,

where $E[\tilde{N}]$ was given in equation (3.18). 
Proof: From (3.1)-(3.3) we get the following expressions for the generating functions of $\left\{\bar{\pi}_{j}\right\}_{j \geq 0}$ and $\left\{\tilde{\pi}_{j}\right\}_{j \geq 1}$

$$
\begin{gathered}
\bar{\Pi}(z)=\sum_{j=0}^{\infty} \bar{\pi}_{j} z^{j}=\bar{\pi}_{0} F\left(1,1+\frac{\lambda+\alpha}{\mu} ; 1+\frac{\alpha+\delta \rho}{\mu} ; \rho z\right) \\
\widetilde{\Pi}(z)=\sum_{j=1}^{\infty} \tilde{\pi}{ }_{j} z^{j}=\frac{\delta(\lambda+\alpha+\mu)}{\nu(\alpha+\mu+\delta \rho) E(\tilde{N})} \rho z F\left(1,2+\frac{\lambda+\alpha}{\mu} ; 2+\frac{\alpha+\delta \rho}{\mu} ; \rho z\right) .
\end{gathered}
$$

Since $(z+1)^{n}=\sum_{k=0}^{n}\left(\begin{array}{l}n \\ k\end{array}\right) z^{k}$, we obtain that $\bar{\Pi}(z+1)=\sum_{k=0}^{\infty} \bar{M}_{k} z^{k}(k !)^{-1}$, and therefore $\bar{M}_{k}$ can be obtained by a direct identification for the $k$ th coefficient of the series $\bar{\Pi}(z+1)$. Thus, we have from the definition of hypergeometric series and (3.24) that $\bar{M}_{k}$, for $k \geq 1$, is given by

$$
\bar{M}_{k}=\bar{\pi}_{0} \sum_{j=k}^{\infty} \frac{\left(1+\frac{\lambda+\alpha}{\mu}\right)_{j}}{\left(1+\frac{\alpha+\delta \rho}{\mu}\right)_{j}} \frac{j !}{(j-k) !} .
$$

By noting that $(1)_{j+k}=(j+k)$ ! and $(a)_{j+k}=(a)_{k}(a+k)_{j}$, it follows that $\bar{M}_{k}$, for $k \geq 1$, is given by (3.22).

Expression (3.23) for $\tilde{M}_{k}$, for $k \geq 0$, can be obtained along the same arguments. By using again the ratio test and Raabe's test it is verified that $\bar{M}_{k}$ and $\mathscr{M}_{k}$ exist for every $k$.

To conclude this section, we summarize in the following corollary some explicit results for the case of constant retrial discipline.

Corollary 1: If $\mu=0, \alpha>0$ and $\gamma<1$, then

i) The probability distribution of the embedded Markov chain at service completion epochs is given by

$$
\bar{\pi}_{j}=\left(1-\frac{\lambda(\lambda+\alpha)}{\nu \alpha+\delta(\lambda+\alpha)}\right)\left(\frac{\lambda(\lambda+\alpha)}{\nu \alpha+\delta(\lambda+\alpha)}\right)^{j}, \text { for } j \geq 0
$$

and its corresponding $k$ th factorial moment is

$$
\bar{M}_{k}=k !\left(\frac{\lambda(\lambda+\alpha)}{\nu \alpha+(\delta-\lambda)(\lambda+\alpha)}\right)^{k}, \text { for } k \geq 0 .
$$

ii) If $\delta>0$, the probability distribution of the embedded Markov chain at killing epochs is given by

$$
\tilde{\pi}_{j}=\left(1-\frac{\lambda(\lambda+\alpha)}{\nu \alpha+\delta(\lambda+\alpha)}\right)\left(\frac{\lambda(\lambda+\alpha)}{\nu \alpha+\delta(\lambda+\alpha)}\right)^{j-1}, \text { for } j \geq 1
$$

and its corresponding $k$ th factorial moment is

$$
\tilde{M}_{k}=\frac{\nu \alpha+(\delta-\lambda)(\lambda+\alpha)}{\lambda(\lambda+\alpha)}\left(\frac{\nu \alpha+\delta(\lambda+\alpha)}{\nu \alpha+(\delta-\lambda)(\lambda+\alpha)}\left(\frac{\lambda(\lambda+\alpha)}{\nu \alpha+(\delta-\lambda)(\lambda+\alpha)}\right)^{k} k !-\delta_{0 k}\right), \text { for } k \geq 0 \text {. }
$$

The proof of this result is trivial from theorems 1 and 2 and thus omitted. However, it should be noted that $\bar{\pi}_{j}=\tilde{\pi}_{j+1}$, for $j \geq 0$.

\section{The Departure Process}

The departure process is defined as the sequence of the times $\left\{\eta_{i}\right\}_{i>0}$ at which customers leave the queueing system after their service completion epochs. The study of $\left\{\eta_{i}\right\}_{i} \geq 0$ is equiva- 
lent to the study of $\left\{\tau_{i}=\eta_{i}-\eta_{i-1}\right\}_{i}>1$. It should be noted that the interval $\tau_{i}$ can be expressed as $\tau_{i}=R_{i}+S_{i}$, for $i \geq 1$, where $R_{i}^{-}$is defined as the server idle period until the arrival of the $i$ th (primary or orbiting) customer and $S_{i}$ is the corresponding service time.

We assume that the system is stable, consequently $\tau_{1}, \tau_{2}, \ldots$ are identically distributed random variables. For convenience of notation we will denote the interval under consideration as $\tau_{1}$.

We next give the joint distribution of $\left(R_{1}, S_{1}\right)$ in terms of its Laplace-Stieltjes transform.

Theorem 3: If the queueing system is ergodic, then the Laplace-Stieltjes transform $\phi(\theta, \omega)=E\left[\exp \left\{-\theta R_{1}-\omega S_{1}\right\}\right]$ is given by

$$
\phi(\theta, \omega)=\frac{\nu}{\omega+\nu}\left(1-\frac{\theta}{\theta+\lambda+\alpha} \bar{\pi}_{0}\left(\frac{\alpha}{\theta+\lambda}+{ }_{3} F_{2}\left(\begin{array}{c}
1,1+\frac{\lambda+\alpha}{\mu}, \frac{\theta+\lambda+\alpha}{\mu} ; \rho \\
1+\frac{\alpha+\delta \rho}{\mu}, 1+\frac{\theta+\lambda+\alpha}{\mu}
\end{array}\right)\right),\right.
$$

for $\operatorname{Re}(\theta) \geq 0, \operatorname{Re}(\omega) \geq 0$, where $\bar{\pi}_{0}$ was given in $(3.1)$.

Proof: Since the length of service time, $S_{1}$, is independent of all the events occurred before its commencement, the random variables $R_{1}$ and $S_{1}$ are independent. Thus,

$$
\phi(\theta, \omega)=E\left[\exp \left\{-\theta R_{1}\right\}\right] E\left[\exp \left\{-\omega S_{1}\right\}\right]
$$

where $E\left[\exp \left\{-\omega S_{1}\right\}\right]=\nu(\omega+\nu)^{-1}$.

In order to find the Laplace-Stieltjes transform of $R_{1}$, we condition on the number of customers present in the system at the previous service completion epoch to get

$$
E\left[\exp \left\{-\theta R_{1}\right\}\right]=\frac{\lambda}{\theta+\lambda} \bar{\pi}_{0}+\sum_{n=1}^{\infty} \frac{\lambda+\alpha+n \mu}{\theta+\lambda+\alpha+n \mu} \bar{\pi}_{n}
$$

Note that

$$
\sum_{n=0}^{\infty} \frac{\bar{\pi}_{n}}{\theta+\lambda+\alpha+n \mu}=\frac{1}{\theta+\lambda+\alpha} \int_{0}^{1} \bar{\Pi}\left(x^{\overline{\theta+\lambda+\alpha}}\right) d x
$$

Thus

$$
\sum_{n=1}^{\infty} \frac{\lambda+\alpha+n \mu}{\theta+\lambda+\alpha+n \mu} \bar{\pi}_{n}=1-\frac{\lambda+\alpha}{\theta+\lambda+\alpha} \bar{\pi}_{0}-\frac{\theta}{\mu} \int_{0}^{1} \frac{\theta+\lambda+\alpha}{t^{\frac{\theta+\lambda}{\mu}}}-1 \bar{\Pi}(t) d t
$$

Substitution of (3.24) into (4.2) leads, after some algebraic manipulations, to (4.1).

Note that the generalized hypergeometric series in (4.1) is convergent if and only if the process $\{X(t), t \geq 0\}$ is ergodic.

In the particular case $\mu=0$ and $\alpha>0$, the above result is reduced to a more explicit expression.

Corollary 2: If $\mu=0, \alpha>0$ and $\gamma<1$, then the Laplace-Stieltjes transform of $\left(R_{1}, S_{1}\right)$ is given by

$$
\phi(\theta, \omega)=\frac{\nu}{(\omega+\nu)(\theta+\lambda+\alpha)}\left(\lambda+\alpha\left(1-\frac{\theta}{\theta+\lambda} \bar{\pi}_{0}\right)\right)
$$

for $\operatorname{Re}(\theta) \geq 0$ and $\operatorname{Re}(\omega) \geq 0$, where $\bar{\pi}_{0}$ was given in corollary 1 .

The Laplace-Stieltjes transform of the pair $\left(R_{1}, S_{1}\right)$ can be inverted by inspection. This 
yields the following result.

Corollary 3: If the process $\{X(t), t \geq 0\}$ is ergodic, then

i) If $\mu=0$ and $\alpha>0$,

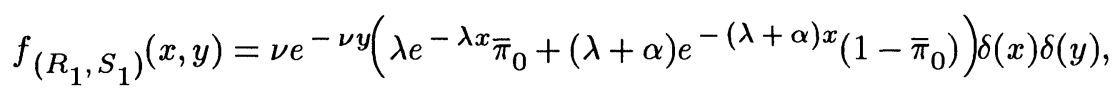

where $\bar{\pi}_{0}$ was given in corollary 1 and $\delta(\cdot)$ denotes Dirac's delta.

ii) If $\mu>0$,

$$
\begin{gathered}
f_{\left(R_{1}, S_{1}\right)}(x, y)=\bar{\pi}_{0} \nu e^{-\nu y}\left(\lambda e^{-\lambda x}+\frac{\lambda+\alpha+\mu}{\alpha+\mu+\delta \rho} \rho e^{-(\lambda+\alpha+\mu) x}\right. \\
\left.\times\left((\lambda+\alpha) F\left(1,2+\frac{\lambda+\alpha}{\mu} ; 2+\frac{\alpha+\delta \rho}{\mu} ; \rho e^{-\mu x}\right)+\mu F\left(2,2+\frac{\lambda+\alpha}{\mu} ; 2+\frac{\alpha+\delta \rho}{\mu} ; \rho e^{-\mu x}\right)\right)\right) \delta(x) \delta(y),
\end{gathered}
$$

where $\bar{\pi}_{0}$ was given in (3.1).

Our next objective is to obtain formulae for the moments of random variable $R_{1}$. Let $M_{k}^{R}=E\left(R_{1}^{k}\right)$, for $k \geq 0$.

Theorem 4: Under ergodicity, the moments of $R_{1}$ are given by

i) If $\mu=0$ and $\alpha>0$, then

$$
M_{k}^{R_{1}}=k !\left(\frac{1-\bar{\pi}_{0}}{(\lambda+\alpha)^{k}}+\frac{\bar{\pi}_{0}}{\lambda^{k}}\right), \quad \text { for } k \geq 0,
$$

where $\bar{\pi}_{0}$ was given in corollary 1 .

ii) If $\mu>0$, then

$$
M_{k}^{R_{1}}=k ! \bar{\pi}_{0}\left(\frac{1}{\lambda^{k}}+\frac{1}{(\lambda+\alpha)^{k}}\left(k+1 F_{k}\left(\begin{array}{c}
1, \frac{\lambda+\alpha}{\mu}, \ldots, \frac{\lambda+\alpha}{\mu} ; \rho \\
1+\frac{\alpha+\delta \rho}{\mu}, 1+\frac{\lambda+\alpha}{\mu}, \ldots, 1+\frac{\lambda+\alpha}{\mu}
\end{array}\right)-1\right)\right),
$$

for $k \geq 1$, where $\bar{\pi}_{0}$ was given in (3.1).

Proof: With the help of (4.3) we easily obtain the expression (4.4). Upon differentiation of (4.1) we also obtain

where

$$
M_{k}^{R}=k !\left(\frac{1}{\lambda^{k}}-\frac{1}{(\lambda+\alpha)^{k}}\right) \bar{\pi}_{0}-(-1)^{k} \frac{1}{\mu} I_{k}(0)
$$

and

$$
I_{k}(\theta)=\frac{d^{k}}{d \theta^{k}}\left(\theta \int_{0}^{1} t^{\frac{\theta+\lambda+\alpha}{\mu}}-1 \bar{\Pi}(t) d t\right)=\theta J(\theta, k)+k J(\theta, k-1),
$$

$$
J(\theta, k)=\frac{d^{k}}{d \theta^{k}}\left(\int_{0}^{1} \frac{\theta+\lambda+\alpha}{t}-1 \bar{\Pi}(t) d t\right)
$$

After some algebraic manipulations we find that

$$
J(\theta, k)=(-1)^{k} k ! \frac{\mu}{(\theta+\lambda+\alpha)^{k+1}} \bar{\pi}_{0 k+3} F_{k+2}\left(\begin{array}{c}
1,1+\frac{\lambda+\alpha}{\mu}, \frac{\theta+\lambda+\alpha}{\mu}, \ldots, \frac{\theta+\lambda+\alpha}{\mu} ; \rho \\
1+\frac{\alpha+\delta \rho}{\mu}, 1+\frac{\theta+\lambda+\alpha}{\mu}, \ldots, 1+\frac{\theta+\lambda+\alpha}{\mu}
\end{array}\right) .
$$


Substituting (4.7) and (4.8) into (4.6), we derive (4.5). Finally, an application of ratio test and Raabe's test guarantees again that the series in (4.5) is convergent if and only if the process $\{X(t), t \geq 0\}$ is ergodic.

To find the $k$ th moment of the interdeparture time $\tau_{1}, M_{k}^{\tau}=E\left[\tau_{1}^{k}\right]$, for $k \geq 0$, we use the simple observation that $R_{1}$ and $S_{1}$ are independent random variables. We thus obtain

where $M_{j}^{S_{1}}=j ! \nu^{-j}$, for $j \geq 0$.

$$
M_{k}^{\tau} 1=\sum_{j=0}^{k}\left(\begin{array}{l}
k \\
j
\end{array}\right) M_{j}^{R_{1}} M_{k-j}^{S_{1}},
$$

In applications, the mean and variance of any performance measure are perhaps the most important quantities. Thus, we give these quantities in the following.

Corollary 4:

i) If $\mu>0$ and $\rho<1$, then

$$
\begin{gathered}
E\left[\tau_{1}\right]=\frac{1}{\nu}+\bar{\pi}_{0}\left(\frac{1}{\lambda}+\frac{\rho}{\alpha+\mu+\delta \rho} F\left(1,1+\frac{\lambda+\alpha}{\mu} ; 2+\frac{\alpha+\delta \rho}{\mu} ; \rho\right)\right) \\
\operatorname{Var}\left[\tau_{1}\right]=\frac{1}{\nu^{2}}+2 \bar{\pi}_{0}\left(\frac{1}{\lambda^{2}}+\frac{\rho}{(\lambda+\alpha+\mu)(\alpha+\mu+\delta \rho)^{3}} F_{2}\left(\begin{array}{c}
1,1+\frac{\lambda+\alpha}{\mu}, 1+\frac{\lambda+\alpha}{\mu} ; \rho \\
2+\frac{\alpha+\delta \rho}{\mu}, 2+\frac{\lambda+\alpha}{\mu}
\end{array}\right)\right) \\
-\left(\bar{\pi}_{0}\left(\frac{1}{\lambda}+\frac{\rho}{\alpha+\mu+\delta \rho} F\left(1,1+\frac{\lambda+\alpha}{\mu} ; 2+\frac{\alpha+\delta \rho}{\mu} ; \rho\right)\right)\right)^{2},
\end{gathered}
$$

where $\bar{\pi}_{0}$ was given in equation (3.1).

ii)

If $\mu=0, \alpha>0$ and $\gamma<1$, then

$$
\begin{gathered}
E\left[\tau_{1}\right]=\frac{\nu^{2} \alpha+\delta(\lambda+\alpha)(\lambda+\nu)}{\lambda \nu(\nu \alpha+\delta(\lambda+\alpha))} \\
\operatorname{Var}\left[\tau_{1}\right]=\frac{1}{\nu^{2}}+\frac{1}{\lambda^{2}}+\frac{1}{(\lambda+\alpha)^{2}}\left(1-\left(\frac{\nu \alpha+(\lambda+\alpha)(\delta+\alpha)}{\nu \alpha+\delta(\lambda+\alpha)}\right)^{2}\right) .
\end{gathered}
$$

\section{The Quasi-Input Process}

Closely connected with the departure process is the quasi-input process. The quasi-input process is defined as the sequence of times $\left\{\xi_{i}\right\}_{i}{ }_{1}$ at which customers begin to be served. Let $\tau_{i}^{\prime}=\xi_{i+1}-\xi_{i}$ be the time-interval between two successive events of this process. It should be noted that $\tau_{i}^{\prime}$ can be expressed in terms of $\tau_{i}^{\prime}=S_{i}+R_{i+1}$, for $i \geq 1$, where the random variables $S_{i}$ and $R_{i+1}$ were defined in the previous section. We continue assuming that the process $\{X(t), t \geq 0\}$ is ergodic. Thus, we can reduce our study to $\tau_{1}^{\prime}=S_{1}+R_{2}$.

We are interested in studying the joint probability distribution of the pair $\left(S_{1}, R_{2}\right)$. Our study is based on the method used in [4] to analyze the process of service commencement epochs in an $M / G / 1$ queue with classical retrial discipline. This approach allows us to obtain the Laplace-Stieltjes transform of $\left(S_{1}, R_{2}\right), \phi^{\prime}(\theta, \omega)=E\left[\exp \left\{-\omega S_{1}-\theta R_{2}\right\}\right]$. In order to obtain $\phi^{\prime}(\theta, \omega)$ we need to define $P_{m}^{(n)}(x)$ as the probability that at epoch $\eta_{1}+$ there are $m$ customers in orbit given that $Q\left(\xi_{1}+\right)=n$ and $S_{1}=x$. In the particular case $\delta=0$, we get 


$$
P_{m}^{(n)}(x)=e^{-\lambda x} \frac{(\lambda x)^{m-n}}{(m-n) !}, \quad \text { for } m \geq n \geq 0 \text { and } x>0 .
$$

Otherwise, we observe that $P_{m}^{(n)}(x)$ is equal to the transient probability at time $x$ that the queue length of the standard $\mathrm{M} / \mathrm{M} / 1$ queue, with arrival rate $\lambda$ and service rate $\delta$, is $m$ given that it is $n$ at time 0 . Thus, we have (see [2])

$$
\begin{aligned}
& P_{m}^{(n)}(x)=\left(1-\frac{\lambda}{\delta}\right)\left(\frac{\lambda}{\delta}\right)^{m}+e^{-(\lambda+\delta) x}\left(\frac{\lambda}{\delta}\right)^{m} \sum_{i=0}^{\infty} \frac{(\lambda x)^{i}}{i !} \sum_{k=0}^{i+m+n+1}(i-k) \frac{(\delta x)^{k-1}}{k !} \\
& +e^{-(\lambda+\delta) x}\left(\frac{\lambda}{\delta}\right)^{\frac{m-n}{2}}\left(I_{m-n}(2 \sqrt{\lambda \delta} x)-I_{m+n+2}(2 \sqrt{\lambda \delta} x)\right)
\end{aligned}
$$

for $m \geq 0, n \geq 0$ and $x>0$, where $I_{r}(z)$ is the modified Bessel function of order $r$ defined as follows

$$
I_{r}(z)=\sum_{i=0}^{\infty} \frac{1}{i !(i+r) !}\left(\frac{z}{2}\right)^{2 i+r}
$$

Because of the random variables $S_{1}$ and $R_{2}$ are not independent, the analysis of the quasiinput process is essentially more complex than the departure process. By extending the arguments given for the model with classical retrials, but with much involved algebraic manipulations, we can obtain the following results.

Theorem 5: If the process $\{X(t), t \geq 0\}$ is ergodic, then we find that

$$
\begin{gathered}
\phi^{\prime}(\theta, \omega)=\sum_{n=0}^{\infty} \bar{\pi}_{n}\left(\lambda A_{n} \sum_{m=0}^{\infty} B_{m}(\theta) p_{m}^{(n)}(\omega+\nu)+\left(1-\lambda A_{n}\right) \sum_{m=0}^{\infty} B_{m}(\theta) p_{m}^{(n-1)}(\omega+\nu)\right), \\
\operatorname{Cov}\left(S_{1}, R_{2}\right)=\sum_{n=0}^{\infty} \bar{\pi}_{n}\left(\lambda A_{n} \sum_{m=0}^{\infty} A_{m} \bar{p}_{m}^{(n)}(\nu)+\left(1-\lambda A_{n}\right) \sum_{m=0}^{\infty} A_{m} \bar{p}_{m}^{(n-1)}(\nu)\right)
\end{gathered}
$$

where

$$
\begin{gathered}
A_{n}=\frac{1}{\lambda+\alpha\left(1-\delta_{0 n}\right)+n \mu} \\
B_{m}(\theta)=\frac{\lambda+\alpha\left(1-\delta_{0 m}\right)+m \mu}{\theta+\lambda+\alpha\left(1-\delta_{0 m}\right)+m \mu} \\
p_{m}^{(-1)}(\omega+\nu)=0, \quad p_{m}^{(n)}(\omega+\nu)=\int_{0}^{\infty} \nu e^{-(\omega+\nu) x} P_{m}^{(n)}(x) d x \\
\bar{p}_{m}^{(-1)}(\nu)=0, \quad \bar{p}_{m}^{(n)}(\nu)=\int_{0}^{\infty} \nu x e^{-\nu x} P_{m}^{(n)}(x) d x
\end{gathered}
$$

for $m \geq 0$ and $n \geq 0$.

The results given in the above theorem provide a theoretical solution for the main problems associated with the quasi-input process. We are specially interested in $\operatorname{Cov}\left(S_{1}, R_{2}\right)$ because $\operatorname{Var}\left[\tau_{1}^{\prime}\right]$ is equal to $\operatorname{Var}\left[\tau_{1}\right]+2 \operatorname{Cov}\left(S_{1}, R_{2}\right)$. Unfortunately, the expressions for $\phi^{\prime}(\theta, \omega)$ and $\operatorname{Cov}\left(S_{1}, R_{2}\right)$ are difficult to simplify with the exception of some particular choices of the parameters $\alpha, \mu$ and $\delta$. In particular, if $\mu=0$ and $\alpha>0$, the quantities $P_{m}^{(n)}(x), p_{m}^{(n)}(\omega+\nu)$ and $\bar{p}_{m}^{(n)}(\nu)$ can be written in terms of hypergeometric series and, consequently, explicit expressions for (5.1) and (5.2) can be obtained. 


\section{Concluding Remarks}

Throughout this paper we have analyzed the departure and quasi-input processes in a singleserver queueing system with linear retrial discipline where, in addition to arriving customers, there are negative arrivals. In the first sections we have described the mathematical model and deduced the probability distributions of the embedded Markov chains at service completion and killing epochs and their corresponding factorial moments. Since the structural form of type $\mathrm{M} / \mathrm{G} / 1$ is not preserved, an approach based on regenerative processes theory has been used to find these distributions. Sections 4 and 5 deal with the study of the departure and quasi-input processes, respectively. The performance measures of the former can be expressed in terms of hypergeometric functions. Most software packets in applied mathematics and statistics include these series among their facilities; thus, we can consider that our results are closed enough. The quasi-input process has more complex structure as compared with the departure process. However, we have summarized the main results related with this performance quantity.

\section{Acknowledgement}

We thank the referee for his/her comments which help us to improve the clarity of the paper. We also thank to Professor G.I. Falin who brought to our attention, during his visit to Madrid, useful methods for developing this paper.

\section{References}

[1] Artalejo, J.R. and Gomez-Corral, A., Quasi-birth and death processes with applications to queues with repeated attempts, (in preparation) 1995.

[2] Conolly, B.W. and Langaris, C., On a new formula for the transient state probabilities for M/M/1 queues and computational implications, J. Appl. Probab. 30 (1993), 237-246.

[3] Cooper, R.B., Introduction to Queueing Theory, Edward Arnold, London 1981.

[4] Falin, G.I., A survey of retrial queues, Queueing Syst. 7 (1990), 127-168.

[5] Farahmand, K., Single line queue with repeated attempts, Queueing Syst. 6 (1990), 223228.

[6] Harrison, P.G. and Pitel, E., Sojourn times in single-server queues with negative customers, J. Appl. Probab. 30 (1993), 943-963.

[7] Martin, M. and Artalejo, J.R., Analysis of an M/G/1 queue with two types of impatient units, Adv. Appl. Probab. 27 (1995), 840-861.

[8] Stidham, Jr., S., Regenerative processes in the theory of queues, with applications to the alternating-priority queue, Adv. Appl. Probab. 4 (1972), 542-577. 


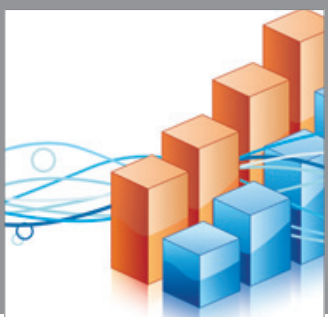

Advances in

Operations Research

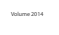

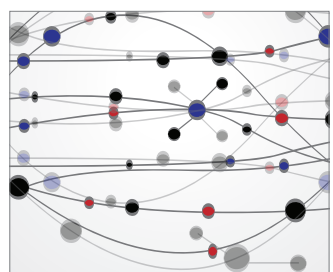

\section{The Scientific} World Journal
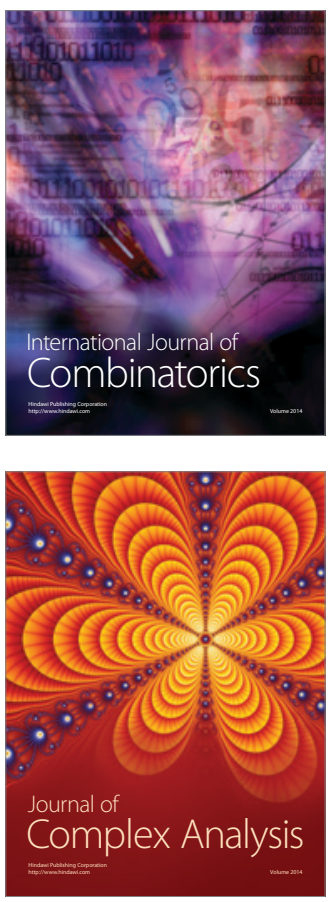

International Journal of

Mathematics and

Mathematical

Sciences
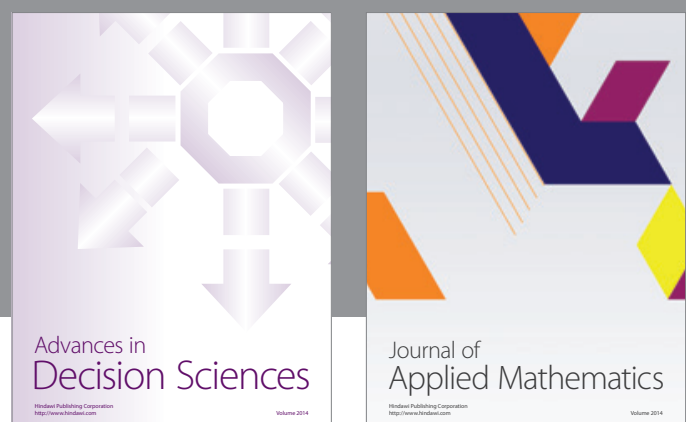

Journal of

Applied Mathematics
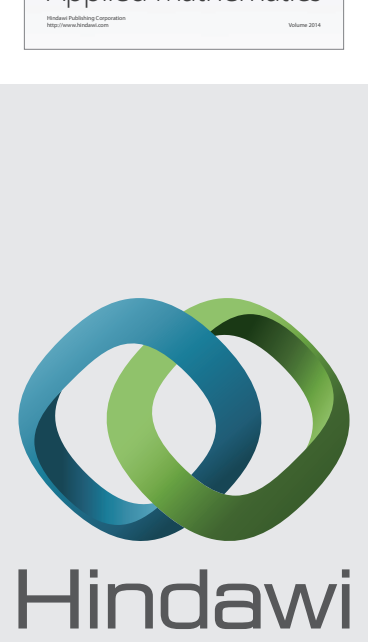

Submit your manuscripts at http://www.hindawi.com
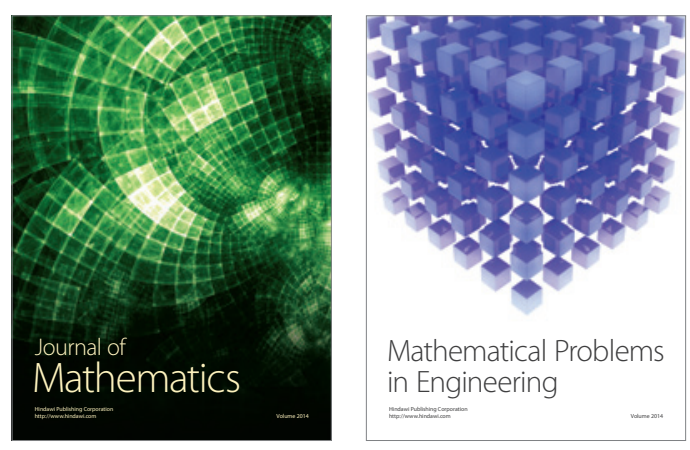

Mathematical Problems in Engineering
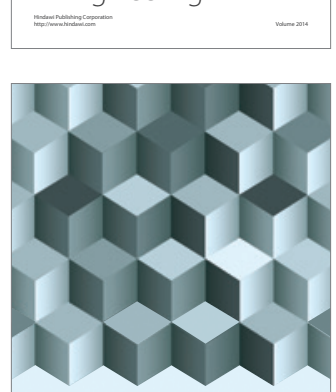

Journal of

Function Spaces
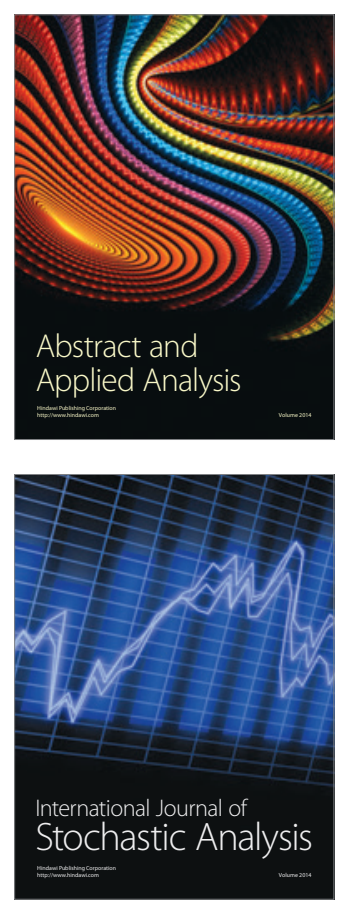

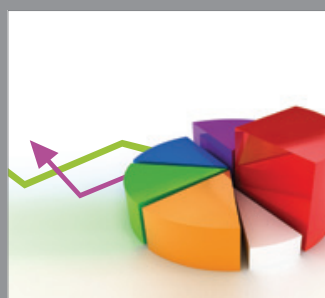

ournal of

Probability and Statistics

Promensencen
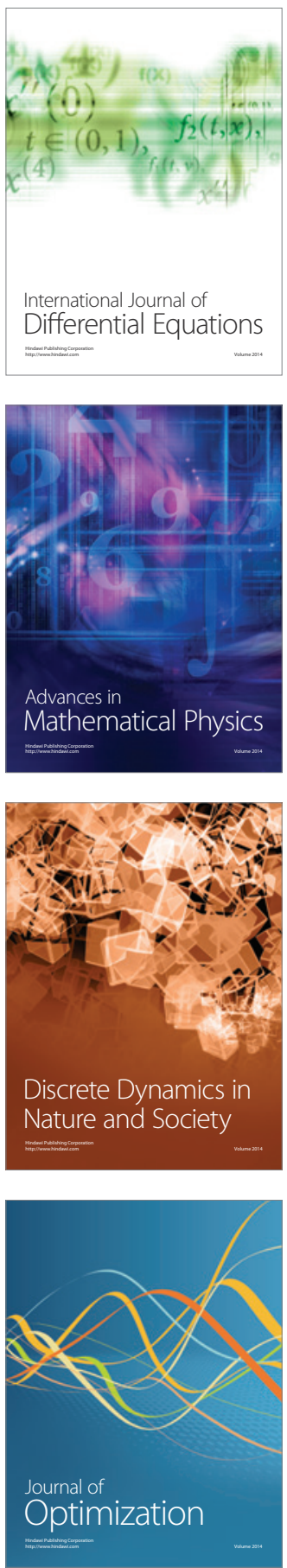